\title{
Experimental Analysis of Energy Efficiency of Server Infrastructure in University Datacenters
}

\author{
Marko SARAC, Sasa ADAMOVIC*, Dusan STAMENKOVIC
}

\begin{abstract}
With the increased number of user applications, the amount of data generated by users, the need for more intensive data processing, in modern data centers the question of energy efficiency arises. IT equipment requires permanent maintenance of appropriate climatic conditions, therefore significant investments are needed in cooling systems and ensuring a constant supply of electricity. In this paper, an experimental analysis is performed concerning the economic and environmental aspects of server virtualization, including the business value of virtualization. An analysis was conducted to be used concurrently on a traditional architecture and a virtual ecosystem. The acquired findings show considerable advantages of virtual and cloud ecosystem in the form of optimum provision and use of physical workstation properties. Through this paper, authors analysed the power utilization when utilizing a higher number of physical servers, as opposed to the same number of virtual servers. Acquired results present a assessment of accumulative energy utilization and load on physical units during optimal workload client requests during the working week. Through the paper authors presented the idea of low electric energy consumption, using the green datacenter concept and contribution to the advancement of IT technologies at the Singidunum University, which is also applicable to other modern university datacenters.
\end{abstract}

Keywords: datacenter; energy efficiency; virtualization

\section{INTRODUCTION}

Computation is increasingly moving to the datacenter. Thus, the energy used by CPUs in the datacenter is gaining importance [1]. In contrast to the traditional method of datacenter organisation, which requires significant financial resources, server virtualization represents the more efficient approach to control the existing assets inside a datacenter. Till 2003, when it was largely considered that virtualization technology first became a stable product (i.e. a solution), around $70 \%$ of the whole virtualization package were associated to development and analysis, including equipment improvement and assessment in the laboratories of sizable enterprises [2, 3].The point up to 2005 also saw a change in the utilization of electric and thermal power [4]. This new permanence and associated package testing conditions steered to the advancement of functions in the field of IT infrastructure, with the aim being on the computer programming of various products aimed at maximising utilisation and reducing both power and cooling costs.

The cost-effective utilization of computer hardware and platforms is manifested in the execution of the servers roles. Varying on the level of processing resource utilisation, datacentres can reach improvements of $20-70 \%$ in terms of electrical power utilization $[5,6]$. In conjunction with virtualization and cloud technology and server merging, datacentres are capable of decreasing the amount of physical machines and maintain the equal level of productivity. This lower energy utilization directly effects in a decrease in operational costs and carbondioxide emissions [7]. The present article compares virtual and physical servers, with experimental measurements revealing the savings that can be made in terms of server energy consumption.

The paper concludes with a discussion regarding the parallels between traditional and virtualised datacentres, including their respective advantages and disadvantages. This paper is divided as follows. Section 2 presents the University datacenter before and after consolidation. Section 3 reports the analysis of experimental measurements. Finally, concluding remarks and future directions are presented in Section 4.4.

\section{UNIVERSITY DATACENTER}

Energy consumption is increasing in the IT sector and a remarkable part of this energy is consumed in datacenters [8]. University datacenters typically comprise many server stations and services, and as a result the IT professionals who manage them are of considerable importance. Although datacenters are often small in size, they remain subject to the problems of cooling and continuous electricity provision. The smooth running of university activities often requires more than 30 machines with various functions and designs [9].

\subsection{The Physical (Traditional) Datacentre}

The key services conducted through the servers on university usually are AD \& DR role, DNS \& DHCP services, firewall, application \& database server, file sharing and file transfer, a system for the electronic testing, a platform for DLS, web hosting and university IS. The physical datacentre consists of seven servers Configuration 1 and five servers Configuration 2, specifications of servers are given below.

To meet all customer requirements, such datacentres require considerable economic investment in terms of hardware, software and infrastructure, in addition to the operational expenses of cooling, management and maintenance.

\subsection{Virtual Architecture Datacenter}

As previously stated, power efficiency can be a great question facing modern Universities and their IT departments. One of the solutions is proposed in the shape of a virtual server built on a power efficient design [10]. As shown in Fig. 1 technology such as virtualization can help in consolidation of server resources, reduction of maintenance complexity and acceleration new 
requirements adoption [11]. Virtual datacentres can also decrease the amount of physical machines, in this case from the existing 12 to 3 physical machines having at least 12 virtual servers. A virtual datacentre consists of three IBM server, specifications of servers are given below.

Diagram of operational university datacentre comprising 12 servers and a diagram of a consolidated and virtualised datacentre is shown in Fig. 1.

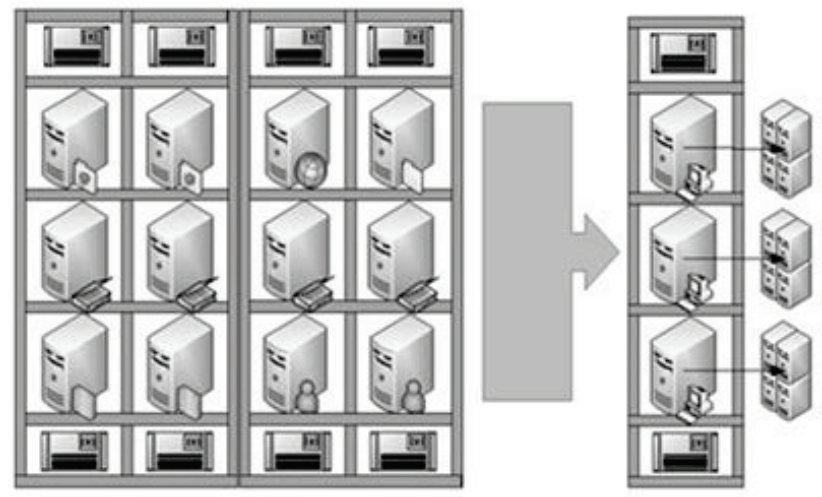

Figure 1 Illustration of University datacenter before and after consolidation

\section{EXPERIMENTAL MEASUREMENTS ANALYSIS}

The results of measurements and analyses are presented in this section. Equipment utilized in the experimental dimensions included:

Three servers based on the Xeon configurations:

First server - Unbranded server, $2 \times$ Intel Xeon 5063, 8 GB $(8 \times 1 \mathrm{~GB})$ FBDIMMDDR2 $667 \mathrm{MHz}, 2 \times 320 \mathrm{~GB}$ HDD, motherboard S5000VSA;

Second server - Unbranded server, $2 \times$ Intel Xeon 5110, 4 GB (4×1 GB) FBDIMMDDR2 $667 \mathrm{MHz}, 2 \times 320$ GB HDD, motherboard S5000VSA;

Third server - IBM x3650 M4, Intel Xeon E5-2620 v2 6C/12T 2.1 GHz $15 \mathrm{MB}, 32 \mathrm{~GB}(4 \times 8 \mathrm{~GB})$ ECC DIMM DDR3 $1600 \mathrm{MHz}, 6 \times 300 \mathrm{~GB}, 1 \mathrm{~TB}$ SAS HDD SATAIII;

A device used in assessing energy utilization and carbon dioxide releases: APC BR900GI.

Software platform on servers: Slackware 13, CentOS 7, Windows Server 2008 R2, Windows 2012 R2. Ed.

Benchmarking software: FinalWire AIDA64 Engineer

Prior to experimental measurement, the required technical specifications of all components and their predicted electricity consumption proposed by the manufacturer were collected. Formula for total energy power consumption in idle state could be based on the model:

$P_{\text {srv_idle }}=P_{\mathrm{CPU}}+P_{\mathrm{RAM}}+P_{\mathrm{HDD}}+P_{\text {other }}$

$P_{\text {srv_idle }}$ represents a total power consumption of the server in the idle state, $P_{\mathrm{CPU}}$ is consumption for processor, $P_{\text {RAM }}$ is the total consumption for all modules of RAM, $P_{\mathrm{HDD}}$ is the total consumption of all hard drives, $P_{\text {other }}$ is the total consumption of all other hardware components such as fans, additional network cards, optical devices, etc. Furthermore, as the servers are usually under constant workload and use a certain portion of the available resources, the formula will be complete if we add the workload on each of the components.

$P_{\text {srv }}=$
$=P_{\text {srv } \_ \text {idle }}+W_{1} \cdot P_{\mathrm{CPU}}+W_{2} \cdot P_{\mathrm{RAM}}+W_{3} \cdot P_{\mathrm{HDD}}+W_{4} \cdot P_{\text {other }}$

This extended formula for total server power consumption contains the separate workload $(W)$ for every hardware component of the server. The workload is believed to be constant with server utilization and maybe able to vary during the business day when workload is increased and during nights when workload is relieved because there are not any users.

Our formula of total consumption for one server is based on the formula from related research [12]:

$P_{\text {srv }}=P_{\text {srv_idle }}+\left(P_{\text {srv_idle }}-P_{\text {srv_idle }}\right) \cdot u_{\text {zone } \_\mathrm{y}}$

where $P_{\text {srv idle }}$ is associated to the maximum energy utilization and $u_{\text {zone_y }}$ is the server operation, a fraction of the highest server workload.

Based on the obtained formula and technical specifications of consumption by the manufacturer, we will get the maximum energy consumption of a server.

Initial experimental measurements have confirmed that the maximum consumption of electricity of a server by the hardware components is slightly larger than the prescribed specification values of hardware manufacturer represented in Tab. 1.

\begin{tabular}{|c|c|c|c|c|}
\hline Hardware component & $\begin{array}{c}\text { Individual } \\
\text { consumption }\end{array}$ & $\begin{array}{c}\text { Number of } \\
\text { pieces }\end{array}$ & $\begin{array}{c}\text { Maximum } \\
\text { consumption }\end{array}$ & $\begin{array}{c}\text { Total } \\
\text { consumption }\end{array}$ \\
\hline Intel $\AA$ Xeon $\AA 5063$ & $95 \mathrm{~W}$ (TDP) & 2 & $190 \mathrm{~W}$ & \multirow{3}{*}{$280.4 \mathrm{~W}$} \\
\hline Kingston ${ }^{\circledR} 1$ GB FBDIMM DDR2 $667 \mathrm{MHz}$ & $9.5 \mathrm{~W}$ & 8 & $76 \mathrm{~W}$ & \\
\hline Western Digital ${ }^{\circ} 320$ GB HDD SATAII & $7.2 \mathrm{~W}$ & 2 & $14.4 \mathrm{~W}$ & \\
\hline Intel $\AA$ Xeon $\AA 5110$ & $65 \mathrm{~W}(\mathrm{TDP})$ & 2 & $130 \mathrm{~W}$ & \multirow{3}{*}{$182.4 \mathrm{~W}$} \\
\hline Kingston ${ }^{\circledR} 1$ GB FBDIMM DDR2 $667 \mathrm{MHz}$ & $9.5 \mathrm{~W}$ & 4 & $38 \mathrm{~W}$ & \\
\hline Western Digital ${ }^{\circledR} 320$ GB HDD SATAII & $7.2 \mathrm{~W}$ & 2 & $14.4 \mathrm{~W}$ & \\
\hline
\end{tabular}

\subsection{Measuring Power Consumption}

In the case of server Configuration 1 power consumption is increased to $21.2 \%$ and to server Configuration 2 increase is $9.1 \%$ higher than cumulative consumption specified by manufacturers; these results are presented in Fig. 2.
During experimental testing, measuring results of electricity consumption in watts are recorded in three different conditions: idle, optimal burden of $40-70 \%$, and fully loaded burden of 90-100\% (fully benchmarked processor, RAM memory and hard disk via AIDA64). These results are presented in Fig. 3, where E1 represents the energy consumption of Configuration 1 server, E2 
energy consumption of Configuration 2 servers and E3 energy consumption for Configuration 3 servers in three different workload modes.

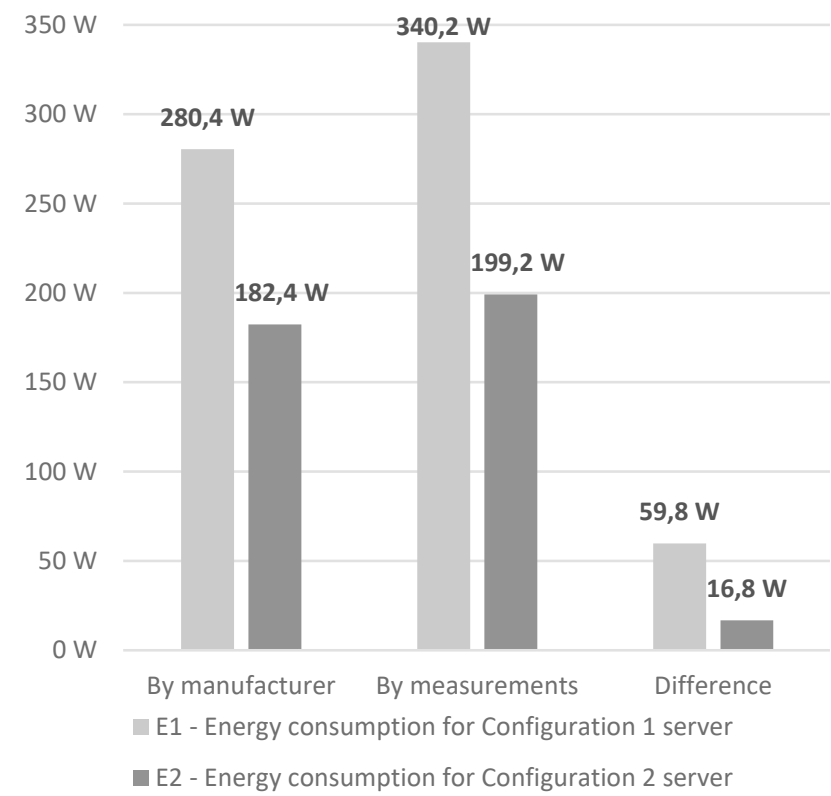

Figure 2 Results of measurements in full benchmark mode

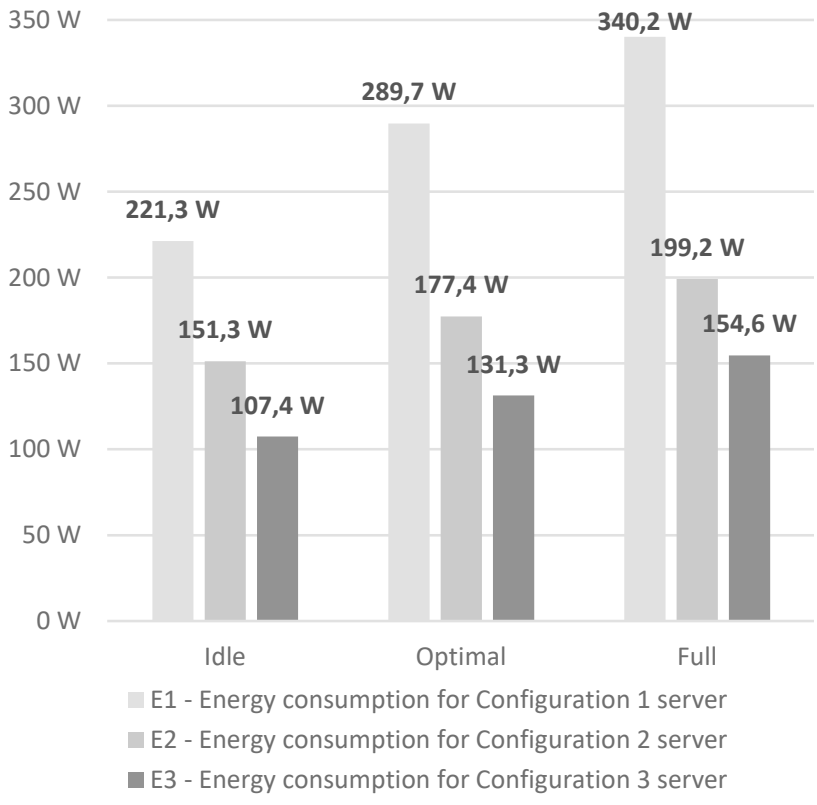

Figure 3 Results of measurements in three workload modes

Values for optimal power consumption for giving servers are the average value of daily consumption followed in the range of 90 days. Results are calculated from measured values obtained from 8 to 20 hours in optimal workload mode and from 20 to 8 hours in idle workload mode for 6 days in the working week and from 0 to 24 hours in idle workload mode for Sunday. Cumulative energy consumption in watts and comparison on daily bases for all three configurations is presented in Fig. 4.

VMware, Citrix and other vendor's software for virtualization is too expensive for Universities in the Republic of Serbia. Because of poor financial situation in our country and facts that Microsoft provides support and donations to educational institutions, Singidunum University is Microsoft's partner that uses Microsoft
Hyper-V for the purpose of virtualization. As we have already stated, traditional datacenter consists of 7 servers Configurations 1 and 5 servers Configuration 2, while virtual datacenter runs 3 servers Configuration 3 . The server consolidation and use of virtualization technology enabled a sixfold reduction in power consumption (Fig. 5). Furthermore, a productional server, replacing up to six physical servers reduces power consumption, additional if we consider the fact that the host machine can himself accomplish some roles. It can be concluded that seven physical servers could be replaced.

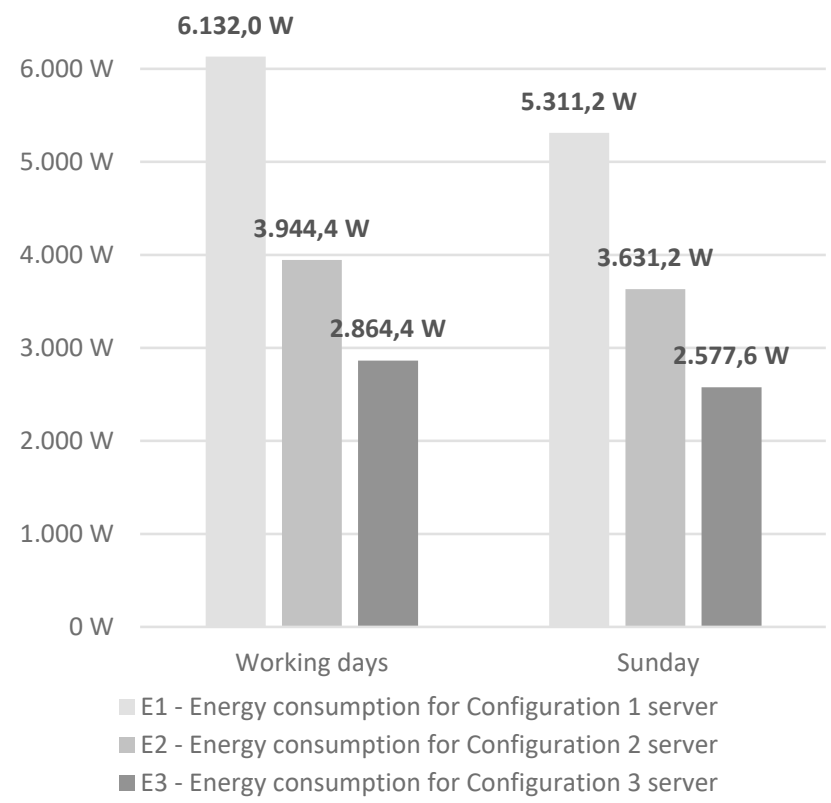

Figure 4 Energy consumption on daily bases

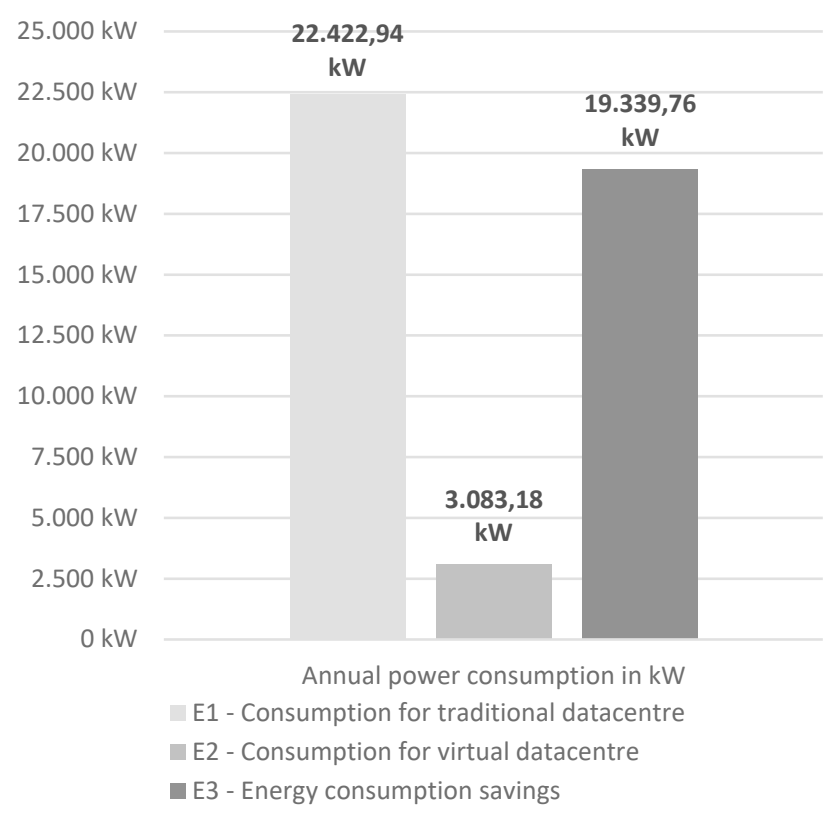

Figure 5 Predicted annual energy consumption savings

To equip labelled machines that are mountable in terms of combining two or four physical CPUs, as well in the ability to boost random access memory up to 2 TB of operational memory, with storage of leading from 8 to 16 units (that can be $500 \mathrm{~GB}$ or larger, up to $4 \mathrm{~TB}$ ), datacentres must initially devote more monetary properties. However, the power reserves available utilizing such servers and 
virtualization package are clear, and thus in the long term their purchase and use will be increasingly cost-effective.

Fig. 6 represents the projected annual savings in the form of monetary properties with a decrease in electricity utilization. Focusing on these results, costs would be reduced almost six times for the given example of consolidation of University datacenter.

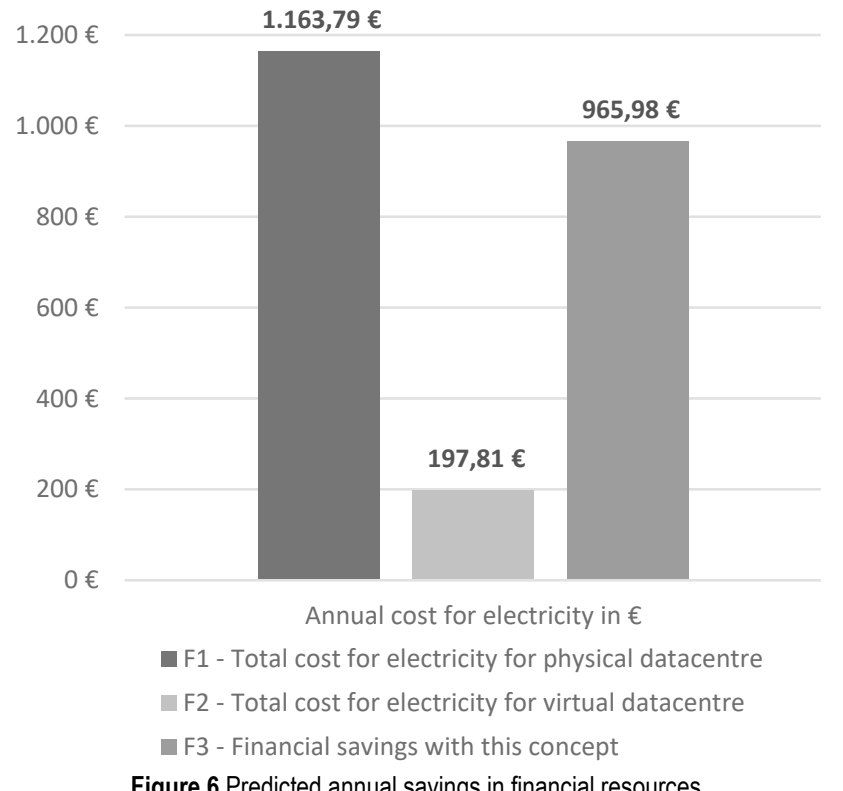

\subsection{Impact on Environment}

In countries of the Balkan region, consciousness, especially about electricity consumption and thus the energy efficiency is at a very low level. The presented paper's social goal is to raise the awareness and to encourage the use of new IT technologies in the preservation of the environment. From the standpoint of environmental protection, reducing electricity consumption would significantly contribute to the reduction of heat release and $\mathrm{CO}_{2}$ emissions (about five times), as shown in (Fig. 7).

Authors Atefeh Khosravi, Saurabh Kumar Garg and Rajkumar Buyya discus this problem in the paper "Energy and Carbon-Efficient Placement of Virtual Machines in Distributed Cloud Data Centers" [16]. Cloud data centers use virtualization technology to host multiple virtual machines (VMs) on a single physical server. By utilizing effective VM deployment processes, cloud service suppliers can improve power efficiency and decrease carbon footprint. Earlier work has concentrated on decreasing energy consumption within one or more data centers regardless of their power resources and energy efficiency (PUE). In contrast, this article recommends a new VM setup algorithm to increase environmental sustainability considering distributed data centers with different carbon footprint rates and PUEs. The model results show that the recommended procedure reduces $\mathrm{CO}_{2}$ emissions and energy consumption, while maintaining the same level of service value compared to other competing systems.

Furthermore paper "An approach to reducing carbon emissions through the migration of virtual machines to a sustainable cloud federation" [17] authored by Maurizio Giacobbe, Antonio Celesti, Maria Fazio, Massimo Villari and Antonio Puliafito discusses a new strategy to reduce carbon dioxide emissions in cloud ecosystems. Specifically, the authors propose a solution that allows providers to determine the best green destination where virtual machines should be relocated to reduce carbon emissions across the federal environment.

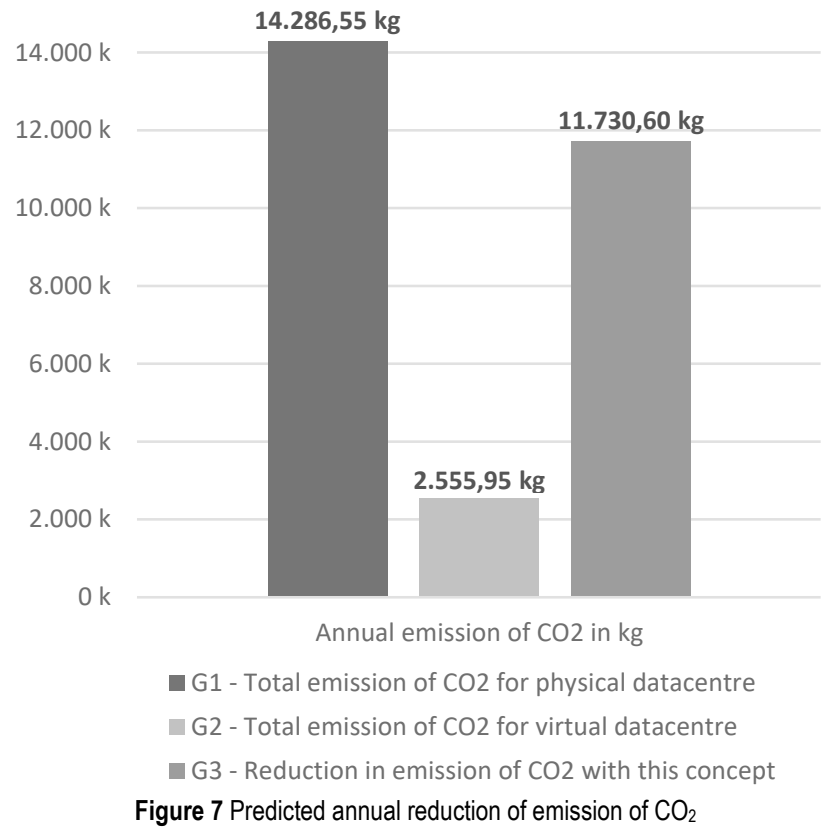

With the consolidation of datacenter using virtualization, cooling devices consume less power to achieve the optimum working temperature in the datacenter because the number of servers is drastically lower. In this way, the additional savings can be represented in the form of decreasing electricity consumption and savings in the financial resources.

\subsection{Business Value of Virtualization and Cloud Computing}

The following section presents an analysis of virtualization for various stages of acknowledgment, with the displayed data comparing the effectiveness of a nonvirtualised enterprise infrastructure and a virtualised environment. The resulting differences between applying virtualization and Cloud Computing are also outlined.

Educational institutions in Serbia are often faced with financial problems. They do nt have the resources for investments in new computer equipment. It often happens that the server infrastructure is not changed even after 10 or more years. The result of this situation is energy inefficient data centers, which are mainly located in inadequately designed and furnished rooms that also consume larger amounts of electricity than necessary.

The concept of virtualization requires a higher initial investment. In the long run it makes significant savings in financial resources in electricity consumption, but also greatly reduces operating costs in terms of reduced need for administrators of server infrastructure. Datacenters protection is easier, faster treatment installations of new services and applications are due to some unforeseen disaster, quickly restoring server infrastructure in 
operational condition are also reasons of adoption of this technology.

Fig. 8 shows further characteristics of the evolution to virtualized services. As anticipated by authors, the physical servers number assigned per manager almost doubles, from 15 in the non-virtualised structure to 27 under virtualized infrastructure. [4]

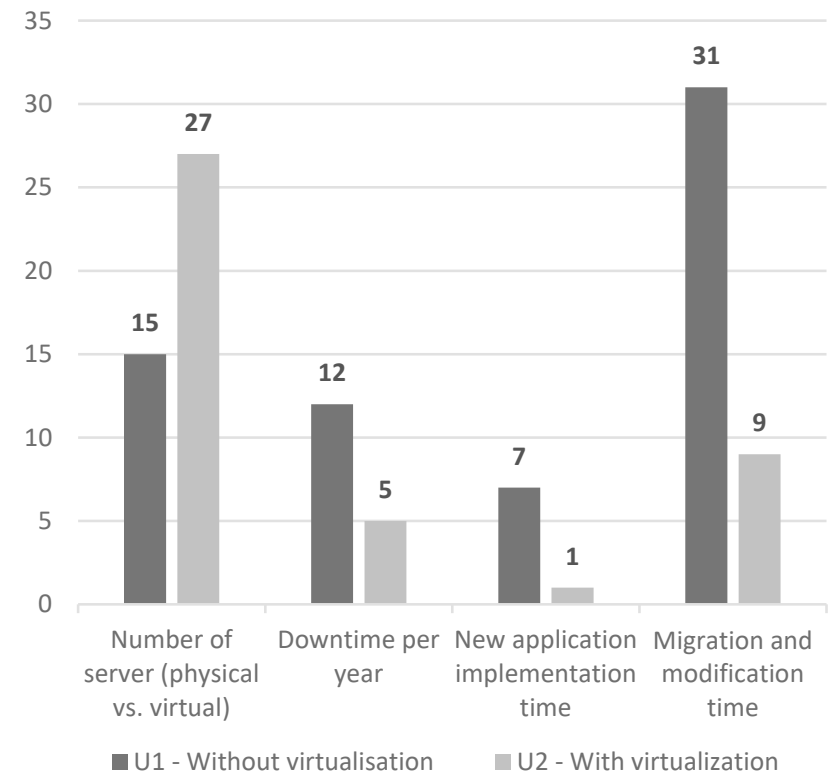

Figure 8 Business value derived by introducing virtualization, experimental analysis

$U 1$ represents datacenter without virtualization with 15 physical servers while $U 2$ represents the virtualised datacenter with 27 mixed physical and virtual servers and also contains the primary enterprise benefit of virtualization components that exceeds the total cost of ownership (TCO). According to the obtained results, the use of virtualised servers decreases annual downtime (in days) and significantly reduces the time required to run new applications (in hours). The time (in hours) required to upgrade and migration is also significantly decreased.

If educational institutions want to contribute to energy efficiency and environmental protection, but do not have enough financial resources for the replacement of all or part of server infrastructure, they can relocate their traditional datacenter to the cloud. Large companies such as Microsoft, can provide ability to use their resources at a much lower price than the market price for educational institutions, or even for free, depending on the need. Microsoft Azure Cloud Platform does not require a large initial investment. A wide range of cloud computing operates on a "pay-as-you use on a monthly basis." The user has access to various services, from ready-made applications such as Office365 (SaaS) via the pre-installed web servers, databases, Active Directory (PaaS) to the renting of the complete server infrastructure (IaaS) based on virtualization.

The cost of monthly maintenance or rent of a server infrastructure may vary from month to month. The reason lies in the configuration of virtual machines, the amount of the realized traffic to and from the service in the cloud, number of accesses, etc.

The advantage of using Infrastructure as a Service Cloud platform compared to traditional or virtual datacenter is reflected in the fact that organizations do not have a local server infrastructure. They do not have to worry about the stable electricity supply, cooling systems, as well as the maintenance by the administrator. It is possible to move a complete datacenter into the cloud. For the administration and utilization of these resources only one obligatory thing is needed - a stable internet connection with the organization. All obligations regarding the maintenance of infrastructure, making security copies (backup), data protection, etc. are implemented by provider, in this case which, according to Microsoft SLA agreement on the use of the Azure platform guarantees $99.9 \%$ availability during the 365 days of the year.

\section{CONCLUSION}

The worldwide energy consumption of data centers increased 56\% between 2005. and 2010., it reached 237 terawatt hours (TWh) in 2010., accounting for over $1 \%$ of the world's electricity usage [13]. It is estimated that the market was worth about 128 billion dollars in 2012, and will grow by about 5 percent yearly to reach 152 billion dollars in 2016 [14]. The presented work is inspired by the current global economic and energy crisis, the search for increased energy efficiency, providing opportunities for both improving IT services and better use of financial resources. To ensure the success of virtualization, it is essential that IT departments take a rational attitude to applicable risks and apply mechanisms to reduce those risks. Though virtualization has genuine advantages, it can also trigger some specific difficulties, particularly for businesses that do not implement the correct administration instruments necessary to handle the new ecosystem. The testing was completed in two different conditions, but involving the use of the identical consumption module.

Experimental analysis revealed that virtual machines (VMs) under light load are consolidated into a small number of physical machines (PMs) to turn idle PMs into low-power states. For system engineers, it means that one physical server can run more virtual servers that are lightly loaded [15]. Virtualization can thus now certainly be considered a mature product in the IT industry, allowing users to create backups as well as enabling hardware independence and optimal utilization.

In the future, virtual datacenter will presumably replace the Cloud datacenter or become hybrid solution. Further research will focus on security mechanisms in protecting data in the cloud, because it is much more difficult to manage the data that are displaced from the datacenter organizations.

\section{Acknowledgement}

This research was supported by the Ministry of Science and Technological Development of the Republic of Serbia through the project TR32054.

\section{REFERENCES}

[1] Mckeown, M., Balkind, J., \& Wentzlaff, D. (2014). Execution Drafting: Energy Efficiency through Computation Deduplication. Proceedings of $4^{\text {th }}$ Annual IEEE/ACM International Symposium on Microarchitecture (MICRO), Cambridge, 432-444. https://doi.org/10.1109/MICRO.2014.43

[2] Orgerie, A., Assuncao, M. D., \& Lefevre, L. (2014). A Survey on Techniques for Improving the Energy Efficiency 
of Large-Scale Distributed Systems. ACM Computing Surveys (CSUR), 46(4), 1-47. https://doi.org/10.1145/2532637

[3] Williams, J. (2012). Private cloud: get it right and exploit its energy economics. Computer Weekly. Retrieved from http://www.computerweekly.com/ehandbook/ Computer-Weekly-Buyers-Guide-to-private-cloud.

[4] Mahesh, S., Trumbach, C., \& Walsh K. (2012). Visualizing technology mining results on life cycle axes: A study of server virtualization. Information Knowledge Systems Management, 11(3-4), 321-343. https://doi.org/10.3233//KS-2012-0213

[5] Kroeker, K. L. (2009). The Evolution of Virtualization. Communications of the ACM, 52(3), 18-20. https://doi.org/10.1145/1467247.1467253

[6] Kleidermacher, D. (2009). Methods and applications of system visualization using Intel visualization technology (Intel VT). Intel Technology Journal, 13(1), 74-83.

[7] Sinha, R. (2009). Green Building: A Step towards Sustainable Architecture. ICFAI Journal of Infrastructure, 7(2), 91-102.

[8] Sharifi, L., Rameshan, N., Freitag, F., \& Veiga, L. (2014). Energy Efficiency Dilemma: P2P-cloud vs. Datacenter. Proceedings of $6^{\text {th }}$ International Conference on Cloud Computing Technology and Science (ClLOUDCOM), Singapore, 611-619. https://doi.org/10.1109/CloudCom.2014.137

[9] Douglas, K. (2010). Through an Event Log. Information Society, 26(1), 65-69. https://doi.org/10.1080/01972240903423394

[10] Bill, A., Anderson, A., Huntley, B., Neiger, G., Rodgers, D., \& Smith, L. (2010). Architected for performancevirtualization support on nehalem and westmere processors. Intel Technology Journal, 14(3), 84-102.

[11] Atkinson, W. (2014). Inside the Data Center Transformation. World Wide Web CIO Insight. Retrieved from http://www.cioinsight.com/it-news-trends/inside-the-datacenter-transformation.html (20.04.2016)

[12] Zhang, X., Lindberg, T., Svensson, K., Mousavi, A., \& Vyatkin, V. (2015). Energy Consumption Modeling of Data Center IT Room with Distributed Air Flow. Proceedings of $20^{\text {th }}$ IEEE International Conference on Emerging Technologies and Factory Automation (ETFA), Luxembourg.

[13] Koomey, J. (2011). Growth in data center electricity use 2005 to 2010. Analytics Press. Retrieved from $\mathrm{http} / / /$ www.mediafire.com/file/zzqna34282frr2f/koomeydat acenterelectuse2011 finalversion.pdf (12.04.2016)

[14] Kovar, J. F. (2014). The 2014 Data Center 100. CRN 2014. Retrieved from http://www.crn.com/news/data-center/ 300071759/the-2014-data-center-100.htm

[15] Akiyama, S., Hirofuchi, T., \& Honiden, S. (2014). Energy Efficiency Dilemma: P2P-cloud vs. Datacenter. Proceedings of $6^{\text {th }}$ International Conference on Cloud Computing Technology and Science (ClLOUDCOM), Singapore, 759762.

[16] Khosravi, A., Garg, S. K., \& Buyya, R. (2013). Energy and Carbon-Efficient Placement of Virtual Machines in Distributed Cloud Data Centers. European Conference on Parallel Processing, Euro-Par 2013: Parallel Processing, 317-328. https://doi.org/10.1007/978-3-642-40047-6_33

[17] Giacobbe, M., Celesti, A., Fazio, M., Villari, M., \& Puliafito, A. (2015). An approach to reduce carbon dioxide emissions through virtual machine migrations in a sustainable cloud federation. Proceedings of International Conference Sustainable Internet and ICT for Sustainability (SustainIT)/IEEE, Madrid, Spain. https://doi.org/10.1109/SustainlT.2015.7101383

\section{Contact information:}

Marko SARAC, PhD, Assistant professor Singidunum University, Danijelova 32, 11000 Belgrade, Serbia E-mail: msarac@singidunum.ac.rs

Sasa ADAMOVIC, PhD, Assistant professor (Corresponding author) Singidunum University, Danijelova 32, 11000 Belgrade, Serbia

E-mail: sadamovic@singidunum.ac.rs

Dusan STAMENKOVIC, MSc, Teaching assistant Singidunum University, Danijelova 32, 11000 Belgrade, Serbia

E-mail: dstamenkovic@singidunum.ac.rs 